\title{
„Schau rein, was für Dich drinsteckt“ - den MTRA-Beruf mit allen Sinnen kennenlernen
}

Im November, dem Entdeckungsmonat der Röntgenstrahlen, veranstalten die Vereinigung Medizinisch-Technischer Berufe in der Deutschen Röntgengesellschaft (VMTB) und der Dachverband für Technologen/-innen und Analytiker/-innen in der Medizin Deutschland. e.V. (dvta) bereits zum 4. Mal gemeinsam die bundesweiten MTRA-Aktionstage. Unter dem Motto „Schau rein, was für Dich drinsteckt“ öffnen Berufsfachschulen gemeinsam mit radiologischen Instituten ihre Türen und geben einen Einblick in die Technik, die die Medizin revolutionierte.

Doch warum sich die Mühe machen? Der Beruf ist in der Öffentlichkeit wenig bekannt, MTRA werden allerorten gesucht und die Bewerberzahlen sind rückläufig. Erklärtes Ziel der MTRA-Aktionstage ist es daher, sich bei Jugendlichen als spannenden Beruf vorzustellen, und das auf besonders anschauliche Weise: „Für die Bewerber sind die Aktionstage eine gute Möglichkeit, hinter die Kulissen zu schauen“, berichtet Anke Ohmstede, Vorsitzende des dvta. „Sie haben die Möglichkeit, in ein radiologisches Institut hineinzukommen, zu sehen, wie Röntgenuntersuchungen durchgeführt werden, was im CT oder im MRT passiert, wie die Bilder aussehen. Man lernt den Beruf mit allen Sinnen kennen, ist direkt vor Ort, kann mit den Menschen sprechen und den Beruf in Aktion erleben - das ist etwas ganz anderes, als eine Berufsinformation zu lesen. Es sind auch viele MTRASchülerinnen und -Schüler an der Durchführung der Aktionstage beteiligt, d.h. die jugendlichen Besucher haben dort die besten Ansprechpartner für ihre Fragen zur MTRA-Ausbildung." Auch Katja Röhr, Vorsitzende der VMTB, ist von dieser Art der Berufsinformati-

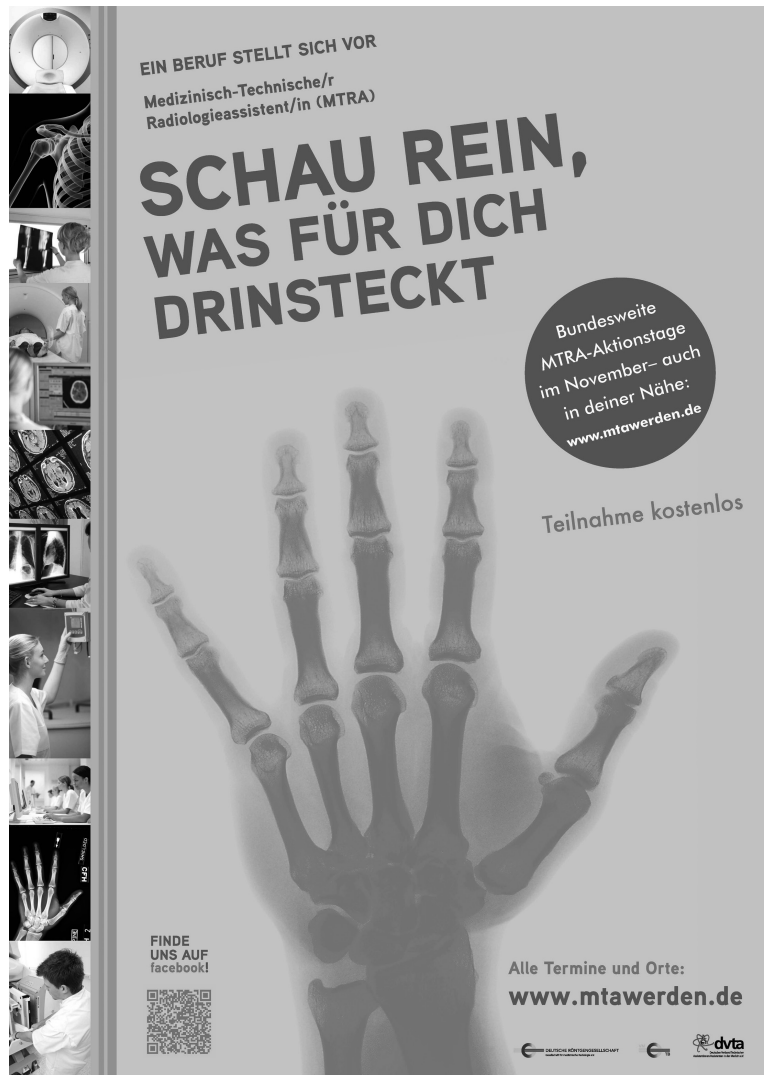


on überzeugt: „MTRA-Schüler können am besten vermitteln, was auf die Interessenten in den nächsten 3 Jahren der Ausbildung zukommt. Zum Beispiel zeigen sie
Gammakameras, fertigen Masken für die Strahlentherapie an, demonstrieren Röntgengeräte und zeigen natürlich auch, wie nach der Ausbildung die berufliche Routi- ne aussieht, z. B. am CT oder MRT. Von einem Aktionstag nimmt der Besucher auf jeden Fall einen bleibenden und umfassenden Eindruck vom MTRA-Beruf mit.“

\begin{tabular}{ll}
\hline $\begin{array}{l}\text { Die MTRA-Aktionstage finden in diesem Jahr an folgenden Standorten statt: } \\
\text { Aachen, 17.11.2012: }\end{array}$ & MTA-Schule am Universitätsklinikum Aachen, Pauwelsstr. 30 \\
\hline Bonn, 10.11.2012: & MTRA-Schule am Universitätsklinikum Bonn, Gebäude 306, Sigmund-Freud-Str. 25, Bonn Venusberg \\
\hline Cottbus, 08.11.2012: & Medizinische Schule an der CTK gGmbH Cottbus, Welzower Str. 27 \\
\hline Fulda, 10.11.2012: & Staatl. anerk. Schule für med.-techn. Assistenten, Carl-Schurz-Str. 14 \\
\hline Hannover, 17.11.2012: & Med. Hochschule Hannover, MTAR-Schule, Bissendorfer Str. 11 \\
\hline Leipzig, 17.11.2012: & Med. Berufsfachschule am Universitätsklinikum Leipzig, Richterstr. 9-11 \\
\hline Mainz, 17.11.2012: & MTAR-Schule an der Universitätsmedizin Mainz, Geb. 505, Zi. E204, Langenbeckstr. 1 \\
\hline Nürnberg, 24.11.2012: & Berufsfachschule für MTRA am Klinikum Nürnberg, Prof.-Ernst-Nathan-Str. 1 \\
\hline Oldenburg, 24.11.2012: & MTA-Schule am Klinikum Oldenburg gGmbH, Brandenburger Str. 19 \\
\hline Stade, 16.11.2012: & 2012 Schule für technische Assistenten in der Radiologie am Elbe Klinikum Stade / Buxtehude, Bremervörder Str. 111 \\
\hline Stuttgart, 20.10.2012: & Klinik für Diagnostische und Interventionelle Radiologie, Kriegsbergstr. 60, Haus E, 1. OG \\
\hline Zwickau, 20.11.2012: & (Allgemeiner Tag der offenen Tür in der Radiologie. Interessierte sind herzlich Willkommen am Infostand der MTA-Studie.) \\
\hline
\end{tabular}

Weitere Informationen zu den MTRA-Aktionstagen 2012 finden Sie unter www.mtawerden.de. 